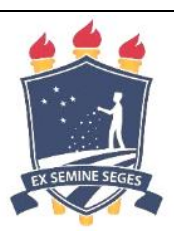

\title{
Avaliação da percepção sobre zoonoses com agentes de saúde, combate a endemias e docentes de escola públicas, do entorno da Estação Ecológica de Caetés, Região Metropolitana do Recife-PE, Brasil
}

\author{
[Perception assessment on zoonoses with health agents, endemic diseases agentes and school teachers \\ public surrounding the Estação Ecológica de Caetés, Metropolitan Region of Recife-PE, Brazil]
}

\section{"Artigo Científico/Scientific Article"}

\author{
George José Alves Costa ${ }^{1 *}$, Sandra Maria Almeida Cavalcanti ${ }^{2}$, Narciso Melo Lins Filho², \\ Samanta Della Bella ${ }^{2}$, Maria Fernanda Vianna Marvulo ${ }^{3,4,5}$, Rozélia Bezerra ${ }^{6}$, \\ Leucio Câmara Alves ${ }^{1}$; Jean Carlos Ramos Silva ${ }^{1,3}$
}

\author{
${ }^{1}$ Departamento de Medicina Veterinária, Universidade Federal Rural de Pernambuco (UFRPE), Recife-PE, Brasil. \\ ${ }^{2}$ Agência Estadual de Meio Ambiente de Pernambuco (CPRH), Unidade de Gestão das Unidades de Conservação, Recife-PE, Brasil. \\ ${ }^{3}$ Instituto Brasileiro para Medicina da Conservação - Tríade, Recife-PE, Brasil. \\ ${ }^{4}$ Faculdade Max Planck, Indaiatuba-SP, Brasil. \\ ${ }^{5}$ Universidade Paulista, Campinas-SP, Brasil. \\ ${ }^{6}$ Departamento de História, Universidade Federal Rural de Pernambuco (UFRPE), Recife-PE, Brasil. \\ *Autor para correspondência/Corresponding author: E-mail: georgejcosta@gmail.com
}

\begin{abstract}
Resumo
Objetivou-se avaliar o aprendizado de professores da disciplina Ciências da Natureza de escolas próximas à Estação Ecológica de Caetés, agentes comunitários de saúde (ACS) e de agentes de combate a endemias (ACE) dos municípios de Abreu e Lima e Paulista, PE, com relação às zoonoses leptospirose, toxoplasmose, raiva e leishmanioses. No primeiro momento foi aplicado um questionário sobre as doenças, tocante ao conceito de zoonoses e sua cadeia epidemiológica. Após as ações de Educação e Saúde foi feito um segundo questionário para verificar o aumento do nível de conhecimento dos participantes. No primeiro questionário cada participante em média, acertou 13 questões a mais do que errou e esta média aumentou para 27 questões mais certas do que erradas no segundo questionário. Em geral, os ACS, ACE e professores possuíam um bom domínio de zoonoses comuns ao seu cotidiano, seja nas campanhas de saúde, ou citadas nas mídias e presentes em meio urbano, como a leptospirose e a raiva. No entanto, em relação às zoonoses negligenciadas como a toxoplasmose e as leishmanioses, os participantes possuíam um nível de conhecimento insuficiente, levando em consideração seu papel de educador. O conhecimento da cadeia epidemiológica da raiva e da leptospirose foi maior do que a toxoplasmose e as leishmanioses. Este trabalho representou a primeira descrição de atividades de educação em saúde sobre zoonoses para ACS, ACE e professores de escolas no entorno de uma Unidade de Conservação no nordeste do Brasil.
\end{abstract}

Palavras-Chave: epidemiologia, educação, SUS, capacitação.

\begin{abstract}
This study aimed to evaluate the learning of teachers of natural sciences schools close to the Ecological Station Caetés, community health agents (CHA) and endemic diseases agents (EDA) of the municipalities of Abreu e Lima and Paulista, $\mathrm{PE}$, about the zoonoses leptospirosis, toxoplasmosis, rabies and leishmaniasis. In the first time was applied a questionnaire on such diseases, concerning the concept of zoonoses and its epidemiological chain. After the actions of health education was answered a second questionnaire to check the increase in the level of knowledge of the participants. In the first questionnaire each participant on average 13 issues hit more than missed and this average increased to 27 more certain than the second erroneous quiz questions. In general, CHA, EDA and teachers had a good knowledge of common zoonoses to their work routine, whether in health campaigns, or quoted in the media and present in urban areas, such as leptospirosis and rabies. However, in relation to neglected zoonoses such as toxoplasmosis and leishmaniasis, participants had insufficient knowledge level, taking into account their role as educators. The Knowledge of the epidemiological chain of rabies and leptospirosis was higher than toxoplasmosis and leishmaniasis. This study represents the first description of health education activities on zoonoses for CHA, EDA and school teachers in the surroundings of a conservation area in northeastern Brazil.
\end{abstract}

Key-words: epidemiology, education, HOS, training. 


\section{Introdução}

As zoonoses são consideradas doenças ou infecções naturalmente transmitidas entre os animais vertebrados (Acha e Szyfres, 1986). O seu conhecimento nem sempre alcança a população exposta aos riscos constantes (Lima et al., 2010) e a educação em saúde pode auxiliar na prevenção e no controle de sua ocorrência nas populações expostas aos riscos. Neste sentido, a educação em saúde constitui de um conjunto de saberes e práticas orientadas para a prevenção de doenças e promoção da saúde (Costa e Lopez, 1996), que por meio de ações pedagógicas constroem cenários de comunicação em linguagens diversas, transformando as informações em dispositivos para o movimento de construção e criação de uma nova realidade (Pedrosa, 2007).

Segundo a Política Nacional de Educação Permanente em Saúde do Brasil, a educação continuada representa uma sucessão do modelo escolar, centralizado na atualização de conhecimentos, com enfoque disciplinar, em ambiente didático e baseado em técnicas de transmissão, com fins de atualização (Brasil, 2009).

No contexto escolar a educação em saúde apesar de ser uma temática multidisciplinar é principalmente abordada nos conteúdos da disciplina 'Ciências da Natureza' e o seu aprendizado nem sempre é de fato ocorrido (Fraga et al., 2009). Neste sentido, as percepções que os professores trazem com relação ao meio ambiente influenciam na sua prática pedagógica e consequentemente na formação dos estudantes, construindo e elaborando uma visão mais crítica, participativa e reflexiva em relação ao mundo que os cerca (Bezerra et al., 2008).

Embora a educação em saúde seja uma das ferramentas da promoção da saúde, tal prática não é muito difundida no sistema de saúde, ressaltandose a necessidade dos profissionais em receberem uma educação permanente que abranjam novas possibilidades metodológicas de atuação (Salci et al., 2013). Sendo assim, o profissional que trabalha com a saúde pública conhece ou precisa conhecer o quão é importante o trabalho dos agentes comunitários de saúde e de endemias, principalmente porque eles são os pilares dos programas de saúde voltados para a atenção básica, instituídos pelo governo, servindo de comunicadores e tradutores desses programas para a população, como um elo entre os outros profissionais e a comunidade (Espínola e Costa, 2006).
Além do mais, os agentes comunitários de saúde (ACS) e agentes de combate às endemias (ACE) representam em média 50\% dos membros de uma equipe de Estratégia Saúde da Família ESF. Na maioria vezes são eles que realizam o maior número de atividades com o objetivo de promover saúde e prevenir agravos a partir de ações educativas (Maciel et al., 2009). Outro ponto importante a ser considerado é a capacitação destes agentes, no sentido de favorecer o desempenho do seu trabalho, por isto ela deverá ser frequente e continuada (Costa et al., 1998; L' Abbate, 1999).

Ao considerar o meio ambiente, existem as Unidades de Conservação que são espaços ambientais que têm importantes características naturais e são legalmente instituídas pelo poder público com objetivos de promover a conservação da vida silvestre. Estes locais possuem limites definidos e existem sob regime especial de administração, ao qual se aplicam garantias adequadas de proteção (Rylands e Brandon, 2005).

De acordo com a Agência Estadual do Meio Ambiente - CPRH, o estado de Pernambuco possui, hoje, 81 Unidades de Conservação Estaduais (40 de Proteção Integral e 41 de Uso Sustentável). Entre as Unidades de Proteção Integral estão 3 Estações Ecológicas, 5 Parques Estaduais e 31 Refúgios da Vida Silvestre e 1 Monumento Natural. Já entre as Unidades de Uso sustentável figuram 18 Áreas de Proteção Ambiental, 8 Reservas de Floresta Urbana e 14 Reservas Particulares do Patrimônio Natural e 1 Área de Relevante Interesse Ecológico (Pernambuco, 2016).

E entre as unidades e proteção integral está a Estação Ecológica de Caetés (ESEC) que possui 157 hectares, localizada na divisa entre os municípios de Paulista e Abreu e Lima.

Levando-se em consideração de que muitos animais silvestres são importantes na cadeia de transmissão de zoonoses, tais como: morcegos na raiva, roedores, marsupiais e carnívoros na leptospirose e nas leishmanioses, felídeos na toxoplasmose, denota-se a importância da formação de recursos humanos quanto a esta temática de suma importância para a Saúde Pública (Silva, 2005). Aliado a isto, os conhecimentos dos professores, ACS e ACE sobre zoonoses como a 'leptospirose', 'toxoplasmose', 'raiva' e 'leishmanioses' precisam ser aprimorados e que tais profissionais necessitem de atualização, pressupõe-se que ações de educação em saúde 
serão úteis para o complemento da formação destes atores.

Diante do exposto, este estudo teve por objetivo avaliar o aprendizado de professores da disciplina Ciências da Natureza, de três escolas próximas à Estação Ecológica de Caetés, município do Paulista, Pernambuco, e avaliar o aprendizado de ACS e ACE dos municípios de Abreu e Lima e Paulista, Estado de Pernambuco, com relação ao conhecimento das zoonoses leptospirose, toxoplasmose, raiva e leishmanioses, por meio de ações de educação em saúde.

\section{Material e Métodos}

A pesquisa foi realizada, por meio de um curso de capacitação para ACS, ACE e professores de escolas dos Municípios de Abreu e Lima e Paulista, Estado de Pernambuco. Os ACS (quatro de Abreu e Lima e três de Paulista) e os 23 ACE (sete de Abreu e Lima e 16 de Paulista) foram selecionados pelos Diretores da Vigilância Epidemiológica das Secretarias Municipais de Saúde. Os cinco professores da disciplina Ciências da Natureza pertenciam a três escolas públicas localizadas no entorno da ESEC, sendo duas escolas de Abreu e Lima e uma de Paulista.

Nas instalações da ESEC foram realizadas as oficinas semanais, com duração de quatro horas onde foram utilizados cartolinas, pincéis atômicos, réguas, pastas de arquivos, lápis grafite, canetas esferográficas, bloco de notas, fitas adesivas, tesouras, colas brancas, panfletos e folders distribuídos em campanhas de saúde, vídeos sobre saúde pública e DVDs com o conteúdo sobre zoonoses.

Esta pesquisa foi dividida em três módulos por meio de oficinas de educação em saúde com intuito de colaborar com o aumento do conhecimento das zoonoses leptospirose, toxoplasmose, raiva e leishmanioses e de suas respectivas cadeias epidemiológicas. Devido à incompatibilidade de horários, os ACS e ACE foram analisados em um período diferente dos professores, porém sob o mesmo método avaliativo.

O primeiro módulo teve como objetivo obter dados para a avaliação do nível de conhecimento dos profissionais sobre os problemas de saúde da comunidade, por meio de um 'diagnóstico participativo'.

Os participantes foram divididos em quatro grupos e cada grupo verificou e analisou um indicador ambiental presente em sua comunidade: grupo 1 - saneamento da água, esgoto e lixo; grupo 2 - controle de cães e gatos errantes; grupo 3 controle de ratos e mosquitos e grupo 4 - outros problemas existentes na comunidade, por exemplo, falta de iluminação pública, segurança, transportes, entre outros.

Em seguida, utilizou-se painéis de cartolina e os membros de cada grupo apresentaram a partir de seus indicadores ambientais, o painel 'a Comunidade que tinham' versus 'a Comunidade que queriam'. Foi ressaltado que todos os participantes seriam os atores e que serviriam como ponte para a transformação da comunidade que tinham para a comunidade que queriam. Com esta dinâmica de grupo foi possível coletar dados para a aplicação de um método de abordagem adaptado à realidade dos participantes.

No segundo módulo foram distribuídos kits formados por pasta de papel, régua, lápis grafite, caneta esferográfica, bloco de notas e duas cópias de um questionário. Este kit tinha o objetivo de fornecer ao participante a oportunidade de criar um próprio material de consulta para futuras ações em educação em saúde.

Com o intuito de obter dados para a avaliação do conhecimento dos ACS, ACE e professores, sobre o conceito e a cadeia epidemiológica das quatro zoonoses estudadas (leptospirose, toxoplasmose, raiva e leishmanioses) aplicou-se o primeiro questionário, antes da explanação sobre zoonoses.

Após a coleta dos questionários, foi ministrada a palestra 'Aprendendo sobre Zoonoses', que abordou sobre a cadeia epidemiológica das quatro zoonoses e durante a apresentação foram dirimidas dúvidas dos participantes sobre o tema. Após a exposição, os participantes responderam o segundo questionário com o mesmo conteúdo do primeiro questionário respondido antes da palestra.

No terceiro módulo objetivou-se criar junto aos participantes diversos tipos de materiais informativos sobre as zoonoses. Por meio de uma palestra foram apresentados os conceitos e as formas de execução das ações de educação em saúde que poderiam ser praticadas pelos profissionais de saúde.

Após a apresentação, os participantes dos quatro grupos anteriormente formados no primeiro módulo, foram divididos para apresentarem um material informativo sobre uma das zoonoses abordadas na capacitação. 
Durante a apresentação de cada grupo, foram relembradas pequenas deficiências em cada material, que deveriam ser observadas na elaboração de um material informativo, como tamanho da fonte, proporção entre os tamanhos dos personagens, cores, entre outras.

O questionário aplicado aos ACS, ACE e professores era estruturado ao todo por 53 questões fechadas, dicotômicas, de múltipla escolha e com cinco questões em escala tipo Likert, com opções que variavam de discordo plenamente até concordo plenamente.

Para a análise dos dados, todas as questões foram agrupadas em dois critérios: 'conhecimento sobre o conceito de zoonose' e 'conhecimento da cadeia epidemiológica da leptospirose, toxoplasmose, raiva e leishmanioses'.

De acordo com o número de questões assinaladas corretamente, os resultados foram divididos nos conceitos 'bom, regular e insuficiente'. No qual, foi calculada e comparada a frequência absoluta e a relativa entre as questões respondidas corretamente pelos ACS, ACE e professores no primeiro questionário, antes das ações de educação em saúde, e o segundo questionário realizado após as ações de educação em saúde.

\section{Resultados}

De maneira geral, nos questionários respondidos pelos ACS, ACE e professores avaliando-se o número de respostas assinaladas corretamente e as assinaladas incorretamente, pôde-se averiguar que, a diferença média entre respostas assinaladas corretamente e as marcadas incorretamente no primeiro questionário foi de 12,7. Já no segundo questionário, observou-se uma diferença média entre as respostas corretas e as incorretas de 27,2. Ou seja, no primeiro questionário cada participante em média, acertou 13 questões a mais do que errou e esta média aumentou para 27 questões mais certas do que erradas no segundo questionário.

$\mathrm{Na}$ tabulação dos dados, a pontuação das respostas corretas dos ACS, ACE, professores e estudantes foi dividida em bom, regular e insuficiente, de acordo com o conhecimento das zoonoses leptospirose, toxoplasmose, raiva $\mathrm{e}$ leishmanioses e de suas respectivas cadeias epidemiológicas. Os resultados dos questionários aplicados com relação ao conhecimento dos ACS, ACE e professores sobre estes dois parâmetros estão descritos na Tabela 1.

Tabela 1. Nível de conhecimento dos agentes comunitários de saúde (ACS), agentes de saúde ambiental e combate às endemias (ACE) e professores sobre as zoonoses leptospirose, toxoplasmose, raiva e leishmanioses. Abreu e Lima e Paulista, 2012.

\begin{tabular}{|c|c|c|c|c|}
\hline \multirow[t]{2}{*}{ Zoonose } & \multicolumn{2}{|c|}{ Conhecimento da Zoonose } & \multicolumn{2}{|c|}{$\begin{array}{c}\text { Conhecimento da Cadeia Epidemiológica da } \\
\text { Zoonose }\end{array}$} \\
\hline & $\begin{array}{c}\text { Primeiro } \\
\text { Questionário* } \\
\text { Acertos / N (\%) }\end{array}$ & $\begin{array}{c}\text { Segundo } \\
\text { Questionário** } \\
\text { Acertos / N (\%) }\end{array}$ & $\begin{array}{c}\text { Primeiro } \\
\text { Questionário* } \\
\text { Acertos / N (\%) }\end{array}$ & $\begin{array}{c}\text { Segundo } \\
\text { Questionário** } \\
\text { Acertos / N (\%) }\end{array}$ \\
\hline \multicolumn{5}{|l|}{ Leptospirose } \\
\hline Bom & $23 / 35(65,7)$ & $27 / 35(77,1)$ & $16 / 35(45,7)$ & $27 / 35(77,1)$ \\
\hline Regular & $12 / 35(34,3)$ & $8 / 35(22,9)$ & $16 / 35(45,7)$ & $8 / 35(22,9)$ \\
\hline Insuficiente & $0 / 35(0,0)$ & $0 / 35(0)$ & $3 / 35(8,6)$ & $0 / 35(0)$ \\
\hline \multicolumn{5}{|l|}{ Toxoplasmose } \\
\hline Bom & $14 / 35(40)$ & $32 / 35(91,4)$ & $8 / 35(22,9)$ & $27 / 35(77,1)$ \\
\hline Regular & $18 / 35(51,4)$ & $2 / 35(5,7)$ & $23 / 35(65,7)$ & $8 / 35(22,9)$ \\
\hline Insuficiente & $3 / 35(8,6)$ & $1 / 35(2,9)$ & $4 / 35(11,4)$ & $0 / 35(0)$ \\
\hline \multicolumn{5}{|l|}{ Raiva } \\
\hline Bom & $24 / 35(68,5)$ & $34 / 35(97,1)$ & $8 / 35(22,9)$ & $33 / 35(94,3)$ \\
\hline Regular & $10 / 35(28,6)$ & $1 / 35(2,9)$ & $20 / 35(57,1)$ & $2 / 35(5,7)$ \\
\hline Insuficiente & $1 / 35(2,9)$ & $0 / 35(0)$ & $7 / 35(20)$ & $0 / 35(0)$ \\
\hline \multicolumn{5}{|l|}{ Leishmaniose } \\
\hline Bom & $9 / 35(25,7)$ & $26 / 35(74,3)$ & $10 / 35(28,6)$ & $13 / 35(37,1)$ \\
\hline Regular & $21 / 35(60)$ & $8 / 35(22,8)$ & $22 / 35(62,8)$ & $18 / 35(51,4)$ \\
\hline Insuficiente & $5 / 35(14,3)$ & $1 / 35(2,9)$ & $3 / 35(8,6)$ & $4 / 35(11,4)$ \\
\hline
\end{tabular}




\section{Discussão}

O uso de atividades de educação em saúde com o intuito de aumentar o conhecimento dos ACS, ACE e professores sobre zoonoses como a leptospirose, toxoplasmose, raiva e leishmanioses nas escolas do entorno de uma Unidade de Conservação é inédito na literatura científica. Vale ressaltar que estas quatro zoonoses são de suma importância visando à promoção da saúde pública no Estado de Pernambuco. Desta forma, estes resultados inéditos não foram obtidos somente pelo fato de se avaliar e capacitar três classes profissionais da mesma forma, mas também pelo método empregado e os resultados atingidos, no qual houve um expressivo aumento do nível de conhecimento das zoonoses pelos ACS, ACE e professores.

No geral a pontuação das respostas corretas dos ACS, ACE e professores foram divididos em bom, regular e insuficiente, de acordo com o conhecimento das quatro zoonoses e de suas respectivas cadeias epidemiológicas (Tabela 1).

Com relação ao conhecimento do conceito de zoonoses, no primeiro questionário, $40 \%$ (14/35) dos ACS, ACE e professores possuíam este conhecimento e no segundo questionário o conhecimento aumentou para 88,6\% (31/35). Fato análogo ao observado por Almeida et al. (2009) cujos resultados obtidos nos questionários aplicados revelaram que apenas 25\% (3/12) dos professores e 29\% (7/24) dos ACS possuíam noções sobre o conceito de zoonoses. De acordo com Tomé et al. (2005) verificou-se que 95,29\% (81/85) das educadoras confirmaram que cães e gatos são reservatórios de zoonoses. Resultado semelhante foi observado por Lima et al. (2010) com pais de alunos de uma escola municipal e uma particular, no bairro de Dois Irmãos, em Recife, Pernambuco no qual 71,8\% (46/64) não sabiam reconhecer o significado do termo zoonoses, porém, $16 \%(25 / 64)$ souberam reconhecer pelo menos um tipo de zoonose.

Para a análise dos participantes decidiu-se realizar o agrupamento das questões corretas com conceitos 'bom', 'regular' e 'insuficiente'. Este agrupamento teve como intuito oferecer uma visão geral e mais completa do nível de conhecimento dos profissionais e estudantes, ao invés de avaliálos por meio de questões individuais, pontuais e específicas. Desta forma, o participante foi avaliado pelo seu conhecimento em questões de situações cotidianas e controversas na aplicação de suas atividades profissionais. Os resultados discutidos a seguir serão referentes ao conceito 'bom' para as quatro zoonoses: leptospirose, toxoplasmose, raiva e leishmanioses.

$\mathrm{Na}$ Tabela 1, observou-se que os itens conhecimento da leptospirose e raiva apresentaram poucas diferenças entre o número de respostas corretas no conceito bom, entre o primeiro e o segundo questionários. Isto foi possivelmente devido ao fato de tais zoonoses serem alvos de diversas campanhas de educação em saúde nas comunidades e os profissionais podem estar habituados a fornecer informações sobre estas doenças. Tais zoonoses foram as mais citadas por pais de alunos entrevistados no estudo de Lima et al. (2010), pois foram as que estavam sob constante vigilância por parte dos serviços de saúde e campanhas de vacinação antirrábica animal que eram realizadas anualmente.

Ademais, o baixo percentual de 22,9\% (8/35) de questões corretas na cadeia epidemiológica da raiva no primeiro questionário e de 94,3\% (33/35) no segundo questionário deveuse possivelmente ao fato de que os agentes e professores possuíam desconhecimento dos ciclos silvestre e rural desta zoonose, pois eles trabalham em áreas urbanas, cujo cão e o gato são os focos das medidas profiláticas das campanhas de vacinação. Resultado também observado por Souza et al. (2008) com relação aos animais suscetíveis à raiva, pois $29 \%(14 / 50)$ dos professores não identificaram os equinos e os bovinos, e $50 \%$ (25/50) não identificaram os suínos como animais susceptíveis. No entanto, aplicando-se em um questionário a pergunta 'bois e cavalos podem atuar como fonte de infecção para o homem' a agentes de saúde e professores, Almeida et al. (2009) verificaram $83 \%(20 / 24)$ de acertos para os agentes de saúde e $75 \%$ (9/12) de acertos para professores.

Todavia, as zoonoses toxoplasmose $\mathrm{e}$ leishmaniose apresentaram um baixo percentual de questões corretas no tocante ao seu ciclo epidemiológico: $22,9 \%(8 / 35)$ e $28,6 \%(10 / 35)$, respectivamente, fato que pode ser sugerido pela negligência destas zoonoses pelo poder público. Consequentemente, os profissionais de saúde, de educação, e a comunidade, não possuem o correto entendimento do ciclo epidemiológico destas duas importantes zoonoses e estes fatores podem ter contribuído para o seu baixo conhecimento. Fato observado por Branco et al. (2012) no município de Maringá, PR que em sua pesquisa incluiu 212 profissionais de saúde e 499 gestantes. Entre os 
médicos $15,1 \%$ (10/66) não sabiam que orientações profiláticas deveriam ser dadas à gestante sobre toxoplasmose. E entre as gestantes, $42,08 \%$ (210/499) afirmaram consumir carne crua e $16,23 \% \quad(81 / 499)$ relataram ter recebido informações sobre prevenção da toxoplasmose durante a gestação. Por isto, torna-se necessário a urgência em melhorar a capacitação de profissionais de saúde envolvidos no atendimento de gestantes em prevenção primária de toxoplasmose.

O baixo número de respostas certas em relação à toxoplasmose inicialmente neste estudo, foi representado de forma semelhante às deficiências encontradas pelos professores dos anos iniciais do ensino fundamental, no município de Araçatuba, SP. Neste município, 80\% (68/85) dos professores incriminavam o cão como disseminador da toxoplasmose e $67,06 \%$ (57/85) desconheciam o assunto. $\mathrm{O}$ papel do gato como transmissor da toxoplasmose foi citado por $92,24 \%$ (79/85) das educadoras, entretanto, 82,35\% (70/85) não souberam dizer de que modo o gato pode disseminar a infecção, e 55,29\% (47/85) desconheciam outras possíveis fontes de infecção do Toxoplasma gondii, além do felino de acordo com Tomé et al. (2005).

Contudo, identificou-se no presente estudo um aumento no percentual de questões corretas sobre o ciclo epidemiológico da toxoplasmose de $22,9 \%$ (8/35) no primeiro questionário para $77,1 \%$ (27/35) no segundo questionário, representando um maior nível de conhecimento desta zoonose. Resultados semelhantes foram alcançados por Almeida et al. (2009), com agentes de saúde e professores, com uma média de acertos de mais de $80 \%$ (29/36), na maioria das questões sobre toxoplasmose.

Nas questões sobre leishmanioses observouse um elevado aumento no percentual de questões corretas quanto ao conhecimento da doença de $25,7 \%$ (9/35) no primeiro questionário para 74,3\% (26/35) no segundo questionário. Em Belo Horizonte, MG qualquer conhecimento sobre a leishmaniose visceral (exceto saber nomes do vetor) foi considerado fator de proteção, capaz de minimizar o risco de ocorrência de leishmaniose visceral em 2,24 vezes (Borges et al., 2008).

No município de Petrolina, PE, dentre os professores avaliados, 79,5\% (35/44) responderam que sabiam o que era o 'calazar' (nome popular para leishmaniose visceral americana, na região), sendo que este conhecimento se restringia ao fato de terem ouvido falar pelos telejornais, ou ainda vivenciado pessoas da família ou estudantes seus acometidos pela enfermidade (Costa et al., 2009).

Fato semelhante foi observado por Gama et al. (1998) com relação aos conhecimentos básicos relativos à Leishmaniose Visceral Americana (LVA) em populações de áreas periurbanas e rurais de três municípios do Estado do Maranhão, no qual 93,8\% (274/293) da população entrevistada somente ouviu falar sobre a LVA por fontes não oficiais. Em Recife, PE, 89,4\% (54/64) dos pais de alunos entrevistados souberam da doença pelos postos de saúde da família (Lima et al., 2010). E na população estudada no município de Mucajaí, RR verificou-se na pesquisa de Gama Neto et al. (2012) que $17,7 \%$ (14/130) dos entrevistados obtiveram informações sobre a leishmaniose com os agentes de saúde.

Contudo, no presente estudo quando questionados sobre o ciclo epidemiológico das Leishmanioses Visceral e Tegumentar, os participantes apresentaram uma pequena diferença entre as respostas corretas de $28,6 \%(10 / 35)$ no primeiro questionário para $37,1 \%$ (13/35) no segundo questionário. Percebeu-se nas respostas dos questionários que os participantes, por exemplo, continuavam a confundir o cão como transmissor da leishmaniose, ao invés do flebotomíneo, necessitando assim de maiores esclarecimentos quanto ao papel do reservatório e do vetor na transmissão das leishmanioses.

Isto pode ser atribuído, talvez, no fato das campanhas de prevenção e controle das leishmanioses serem centradas no cão, como 'culpado' pela zoonose ao ser humano. E, consequentemente, esta falsa premissa leva as pessoas a disseminarem mitos para a população, sendo muito difíceis de serem corrigidos em pouco tempo, pois ficam incrustados no conhecimento popular.

Da mesma forma, Almeida et al. (2009) verificaram com agentes de saúde e professores, que os mesmos possuíam pouca informação sobre a leishmaniose. Gama Neto et al. (2012) verificaram que 43,8\% (57/130) dos entrevistados não sabiam quem transmite a LTA e $55,4 \%$ (72/130) achou que o transmissor é um mosquito, porém não soube determinar exatamente que tipo de mosquito. Gama et al. (1998) em pesquisa semelhante, evidenciaram que 94,9\% (277/293) dos entrevistados não tomavam conhecimento sobre o nome do flebotomíneo que transmitia a leishmaniose. 
Em uma pesquisa realizada no município de Petrolina, PE, Costa et al. (2009) realizaram entrevistas com professores do ensino fundamental de escolas públicas, e ao perguntar "quem conhece um mosquito chamado mosquito-palha, birigui ou cangalinha?" 84,1\% (37/44) dos entrevistados não conheciam o flebótomo, nem mesmo por outro nome. Além disto, ao questionar se "você sabe quem transmite o calazar ou leishmaniose visceral?" $56,8 \% \quad(25 / 44)$ dos professores afirmaram saber a forma de transmissão do calazar ou LVA, entretanto asseguravam também que o cão era o responsável pela transmissão da doença. Fato semelhante com relação a participação do cão na transmissão da LVA foi observado por Gama et al. (1998) que constataram que 87,2\% (255/293) da população analisada reconheceram o envolvimento do cão na cadeia epidemiológica da doença. A respeito da leishmaniose, 91,76\% (78/85) das pessoas afirmaram que o cão é o principal reservatório da Leishmania spp. e 58,82\% (50/85) desconheciam a forma de transmissão da doença. Foi constatado também que 37,65\% (32/85) relataram que o mosquito é o vetor desta enfermidade e 3,53\% (3/85) citaram que a saliva canina é infectante (Tomé et al., 2005).

Com relação a execução de medidas preventivas para a leishmaniose $74,6 \%$ (97/130) dos moradores da vila do Apiaú, município de Mucajaí, RR não faziam o seu uso (Gama Neto et al., 2012). Em relação ao controle da leishmaniose, $60 \%$ (51/85) dos professores de escolas municipais do ensino infantil de Araçatuba, SP citaram como profilaxia desta zoonose a limpeza ambiental (Tomé et al., 2005). Foi possível estimar diminuição de 1,94 vezes o risco de ocorrência de leishmaniose visceral para pessoas que declararam tomar qualquer atitude preventiva, quando comparadas com pessoas que não praticam tais ações em Belo Horizonte, MG (Borges et al., 2008).

No geral, observa-se que nestas pesquisas relacionadas com educação em saúde de zoonoses não foram utilizados ACS, ACE e professores próximos de Unidades de Conservação, principalmente analisando o seu conhecimento bem como na construção de 'uma comunidade que queremos' com foco na melhoria da promoção da saúde. Desta forma, as atividades de educação em Saúde com intuito de envolver os profissionais de saúde (ACS e ACE) e educação (professores) tomando como base a sua realidade e inserção social, cultural e ambiental representou um fundamental recurso para ações de saúde pública visando à promoção da saúde.

As atividades de educação em saúde ressaltando-se o curso de capacitação 'educação e saúde em zoonoses' oferecido aos ACS, ACE e professores, as palestras ministradas e os folders informativos sobre zoonoses serviram como importantes instrumentos didáticos na execução das atividades. Da mesma forma, comprovaram a eficácia na melhoria do conhecimento das zoonoses quatro estudadas, resultados observados no aumento do número de respostas corretas assinaladas no segundo questionário, após as atividades de educação em saúde.

\section{Conflito de Interesse}

Os autores declaram não existir conflito de interesse.

\section{Comitê de Ética}

O presente estudo foi submetido e aprovado no Comitê de Ética e Pesquisa da Universidade de Pernambuco - UPE, sob o $\mathrm{n}^{\circ}$ CAAE: 18733413.2.0000.5207, parecer $\mathrm{n}^{\circ} 382.151$ de 03/09/2013 seguindo-se todas as orientações da resolução 196/96 do Conselho Nacional de Saúde, inclusive com a assinatura de cada participante da pesquisa do Termo de Consentimento Livre e Esclarecido.

\section{Agradecimentos}

À Fundação de Amparo de Ciência e Tecnologia de Pernambuco - (FACEPE), pelo apoio financeiro e as Prefeituras dos municípios de Abreu e Lima e Paulista, à Agência Estadual do Meio Ambiente (CPRH) do Governo do Estado de Pernambuco, à Estação Ecológica de Caetés (ESEC Caetés), ao Instituto Brasileiro para Medicina da Conservação - Tríade e à Secretaria de Educação do Estado de Pernambuco pela participação no planejamento e execução deste trabalho. Leucio C. Alves e Jean C. R. Silva recebem Bolsa de Produtividade do Conselho Nacional de Desenvolvimento Científico e Tecnológico - CNPq.

\section{Referências}

Acha, P.N.; Szyfres, B. Zoonosis y enfermedades transmisibles comunes al hombre y a los animales. 2. ed. Washington: Organization Panamericana de La Salud, Publicación Científica n. 503, 1986. 989 p. 
Almeida, J. C.; Bollmann, C.; Marinho, A.P; Skalski, J.; Amaral, C.H.; Biondo, A.W.; Svoboda, W.K; Wouk, A.F.P.F. Percepção sobre zoonoses de professores e Agentes Comunitários de Saúde do Município de Quatro Barras - PR. Higiene Alimentar, 23: 602-603, 2009.

Bezerra, T.M.O.; Feliciano, A.L.P.; Alves, A.G.C. Percepção ambiental de alunos e professores do entorno da Estação Ecológica de Caetés Região Metropolitana do Recife-PE. Revista Biotemas, 21(1): 147-160, 2008.

Borges, B.K.A.; Silva, J.A.D.; Haddad, J.P.A.; Moreira, E.C.; Magalhães, D.F.D.; Ribeiro, L.M.L., Fiúza, V.D.O.P. Avaliação do nível de conhecimento e de atitudes preventivas da população sobre a leishmaniose visceral em Belo Horizonte, Minas Gerais, Brasil. Cadernos de Saúde Pública, 24(4): 777-784, 2008.

Branco, B.H.M.; Araújo, S.M.; FalavignaGuilherme, A.L. Prevenção primária da toxoplasmose: conhecimento e atitudes de profissionais de saúde e gestantes do serviço público de Maringá, estado do Paraná. Revista Scientia Medica, 22(4): 185-190, 2012.

BRASIL. Departamento de Vigilância Epidemiológica. Guia de vigilância epidemiológica. 7. ed. Brasília: Ministério da Saúde, 2009. 816 p.

Costa, G.J.A.; Ramos, R.A.N.; Pimentel, D.S.; Faustino, M.A.G. Alves, L.C. Educação em saúde em Petrolina: ensino-aprendizagem sobre o Calazar ou Leishmaniose Visceral Americana. In: JORNADA DE ENSINO, PESQUISA E EXTENSÃO, 11., 2009, Recife. Anais... Recife, 2009. p. 199.

Costa, I.C.C.; Unfer, B.; Oliveira, A.G.R.C. Investindo na promoção de saúde: Programas de Saúde da Família (PSF) e Agente Comunitário de Saúde (PACS), uma análise de suas performances em oito municípios brasileiros. Revista Aboprev, 1(1): 30-37, 1998.

Costa, M.; Lopez, E. Educación para la salud. Madrid: Pirámide, p. 25-58,1996

Espínola, F.D.S.; Costa, I.C.C. Agentes comunitários de saúde do PACS e PSF: Uma análise de sua vivência profissional. Revista de Odontologia da Universidade Cidade de São Paulo, 18(1): 43-51, 2006.
Fraga, L.S.; Cardoso, K.M.; Pfuetzenreiter, M.R. As práticas docentes e abordagem sobre zoonoses no ensino fundamental. In: Encontro Nacional de Pesquisa em Educação em Ciências. 7., 2009. Florianópolis. Anais... Florianópolis, Abrapec. 2009.

Gama Neto, J.L.; Paulo, C.S.; Passos, M.A.B. Nível de conhecimentos sobre leishmaniose tegumentar americana entre moradores da vila do Apiaú, município de Mucajaí, Roraima, Brasil. Revista Ambiente: Gestão e Desenvolvimento, 4(1): 59-68, 2012.

Gama M.E.A.; Sousa Barbosa, J.; Pires, B.; Cunha, A.K.B.; Freitas, A.R.; Ribeiro, I.R.; Costa, J.M.L. Avaliação do nível de conhecimento que populações residentes em áreas endêmicas têm sobre leishmaniose visceral, estado do Maranhão, Brasil. Cadernos de Saúde Pública, 14(2): 381-90, 1998.

L'Abbate, S. Educação e serviços de saúde: avaliando a capacitação dos profissionais. Cadernos de Saúde Pública, 15(2): 15-27, 1999.

Lima, A.M.A.; Alves, L.C.; Faustino, M.A.D.G.; Lira, N.M.S.D. Percepção sobre o conhecimento e profilaxia das zoonoses e posse responsável em pais de alunos do préescolar de escolas situadas na comunidade localizada no bairro de Dois Irmãos na cidade do Recife (PE). Ciência e Saúde Coletiva, 15(1) 1457-1464, 2010.

Maciel, M.E.D.; Oliveira Borges, P.K.; Moura Sales, C.; Renovato, R.D. Educação em saúde na percepção de agentes comunitários de saúde. Cogitare Enfermagem, 14(2): 340345, 2009.

Pedrosa, J.I.S. Educação Popular no Ministério da Saúde: identificando espaços e referências. In: BRASIL. Ministério da Saúde. Caderno de Educação Popular e Saúde. Brasília: Ministério da Saúde, p. 13-17, 2007.

PERNAMBUCO. Agência estadual de meio ambiente e recursos hídricos-CPRH. Unidades de Conservação. Disponível em: < http://www.cprh.pe.gov.br/Unidades_de_Con servacao/descricao_das_unidades/41788\%3B 48981\%3B5001\%3B0\%3B0.asp> Acesso em: 02 mai. 2016.

Rylands, A.B.; Brandon, K. Unidades de conservação brasileiras. Megadiversidade, v.1, n.1, p.27-35, 2005.

Salci, M.A.; Maceno, P.; Rozza, S.G.; Silva, D.M.G.V.; Boehs, A.E.; Heidemann, I.T.S.B. 
Educação em Saúde e suas perspectivas teóricas: algumas reflexões. Texto Contexto Enfermagem, 22(1): 224-230, 2013.

Silva, J.C.R. Biodiversidade e saúde. In. Franke C.R.; Rocha, P.L.B.; Klein, W.; Gomes, S.L. Mata Atlântica e biodiversidade. 1. ed. Salvador: Edufba, 2005. Cap. 5, p. 191-219.

Souza, P.; Silva, M.; Pierre, E.; Oliveira, E.; Paploski, I. Educação para crianças do ensino fundamental na prevenção da raiva transmitida por morcegos em Guaraqueçaba,
Paraná. In: CONGRESSO BRASILEIRO DE MEDICINA VETERINÁRIA. 35., 2008, Gramado. Anais... Gramado, 2008. p. 6.

Tomê, R.O.; Serrano, A.C.M.; Nunes, C.M.; Perri, S.H.V.; Bresciani, K.D.S. Inquérito epidemiológico sobre conceitos de zoonoses parasitárias para professores de escolas municipais do ensino infantil de AraçatubaSP. Revista Ciência e Extensão, 2(1): 1-11, 2005. 